\title{
Tyrame [N-(3-hidroxi-1:3:5(10)-estratrien-17ß-il)-4-hidroxi- fenetilamina], aminoestrógeno antitrombótico que disminuye la formación de microvesículas
}

\author{
Mirthala Flores-García', Juan M. Fernández-González², Mireille León-Martínez², \\ Simón Hernández-Ortega², José R. Hernández-López¹,3, Daniela Reyes-Munguía', \\ Hugo Sánchez-Sarabia1, Zeuz Piña-Fragoso ${ }^{1}$ y Aurora de la Peña-Díaz ${ }^{1,3 *}$ \\ 'Departamento de Biología Molecular, Instituto Nacional de Cardiología Ignacio Chávez; ${ }^{2}$ Instituto de Química, Universidad Nacional Autónoma de \\ México; ${ }^{3}$ Departamento de Farmacología, Facultad de Medicina, Universidad Nacional Autónoma de México. Ciudad de México, México
}

\section{Resumen}

Antecedentes: Los estrógenos empleados como anticonceptivos o en la terapia de sustitución se asocian a un incremento en el riesgo de desarrollar trombosis, principalmente durante el primer año de tratamiento y en mujeres con factores de riesgo asociados. Objetivo: Sintetizar, caracterizar e identificar el efecto anticoagulante, antiagregante plaquetario y reductor de las microvesículas del nuevo aminoestrógeno tyrame. Material y métodos: Se emplearon ratones cepa CD1 a los que se les administró por vía subcutánea tyrame $(0,1$ y $2 \mathrm{mg} / 100 \mathrm{~g})$. A las $24 \mathrm{~h}$ se tomó una muestra de sangre para determinar el tiempo de coagulación en sangre total, la concentración de microvesículas y el efecto inhibidor de la agregación plaquetaria. Resultados: El tiempo de coagulación en sangre se incrementó hasta 1.5 veces con respecto al control. La inhibición de la agregación plaquetaria tuvo diferente magnitud dependiendo del agente agonista, siendo completa con colágena. Ambos efectos siguieron una relación dependiente de la dosis. Las microvesículas disminuyeron hasta seis veces con respecto al control. Conclusiones: el tyrame disminuye la agregación plaquetaria y la formación de microvesículas, lo que acentúa su posible utilidad terapéutica como un estrógeno sin efectos trombóticos.

PALABRAS CLAVE: Tyrame. Aminoestrógenos. Trombosis. Microvesículas.

Tyrame [N-(3-hydroxy-1:3:5(10)-estratrien-17 $\beta$-yl)-4-hydroxy-phenethylamine], antithrombotic aminoestrogen that decreases microvesicle formation

\begin{abstract}
Background: Estrogens that are used as contraceptives or in replacement therapy are associated with an increase in the risk for developing thrombosis, mainly during the first year of treatment and in women with associated risk factors. Objective: To synthesize, characterize and identify the anticoagulant, antiplatelet aggregation and microvesicle-reducing effect of the new aminoestrogen Tyrame. Material and methods: CD1 strain mice were used, which had Tyrame $(0,1$ and $2 \mathrm{mg} / 100 \mathrm{~g})$ subcutaneously administered. At $24 \mathrm{~h}$, a blood sample was obtained to determine whole-blood clotting time, microvesicles concentration and inhibitory effect on platelet aggregation. Results: Blood clotting time increased up to 1.5 times in comparison with the control. Platelet aggregation inhibition had different magnitude depending on the agonist agent employed, and was complete with collagen. Both effects had a dose-dependent relationship. The microvesicles decreased up to six times with respect to the control. Conclusions: Tyrame reduces platelet aggregation and microvesicle formation, which emphasizes its potential therapeutic utility as an estrogen free of thrombotic effects.
\end{abstract}

KEY WORDS: Thyrame. Aminoestrogens. Thrombosis. Microvesicles.

Correspondencia:

*Aurora de la Peña-Díaz

E-mail: aurorad@unam.mx
Disponible en PubMed Gac Med Mex. 2021;157:608-612 www.gacetamedicademexico.com 0016-3813/@ 2021 Academia Nacional de Medicina de México, A.C. Publicado por Permanyer. Este es un artículo open access bajo la licencia CC BY-NC-ND (http://creativecommons.org/licenses/by-nc-nd/4.0/). 


\section{Introducción}

Millones de mujeres utilizan estrógenos, solos o en combinación con progestágenos como anticonceptivos orales 0 en terapias de reemplazo hormonal ${ }^{1}$. Sin embargo, diferentes estudios no muestran beneficio para prevención secundaria de la enfermedad coronaria en mujeres ${ }^{2,3}$.

Los estrógenos exógenos no confieren una protección cardiovascular y se asocian a un incremento en el riesgo de desarrollar trombosis venosa y/o arterial, principalmente durante el primer año de tratamiento en mujeres con factores de riesgo asociados, como una mutación en el gen de protrombina $\mathrm{G} 20210 \mathrm{~A}^{4} \mathrm{y}$ factor $V$ Leiden ${ }^{5}$.

Los estrógenos alteran varios de los mecanismos hemostásicos, generando un estado protrombótico ${ }^{6}$.

Las plaquetas activadas concentran sobre los fosfolípidos negativos de su membrana al factor tisular (FT) y los factores de la coagulación circulantes, de esta manera facilitan las interacciones entre ellos generando trombina.

Hasta hace unas décadas se asumía que la función de las plaquetas concluía después de activarse, actualmente se tiene evidencia de que después de la activación y/o de la apoptosis, las plaquetas y otras células forman microvesículas (MV); son fragmentos de membranas que carecen de simetría y una característica importante es que exponen fosfatidilserina y FT sobre su superficie. El fragmento de membrana adopta una forma circular, tienen un tamaño que puede variar entre 100 y 1,000 nm, guardan características de las células de las cuales se forman y por la circulación pueden alcanzar territorios que, en algunos casos, podrían ser inaccesibles para las células que las generan. Recientemente se señala la participación de las MV en muchos estados patológicos, incluyendo las trombosis tanto arteriales como venosas.

Entre las particularidades de las MV destaca su posibilidad de transportar entre la célula que le da origen y otras células miARN, ARNm, ADN, proteínas e iniciar respuestas en los tejidos a los que acceden como es la fosforilación de Akt y expresión de óxido nítrico sintasa endotelial (eNOS).

Las MV se encuentran en un punto angular en el equilibrio fisiológico de la hemostasia. Su actividad puede ser protrombótica si en su estructura portan $\mathrm{FT}^{7}$ y/o profibrinolítica ${ }^{8}$ si en su estructura portan activadores de plasminógeno. Ambos mecanismos explican el concepto del FT y la plasmina que se forman en la sangre y que han dado un giro a los conceptos tradicionales de coagulación y fibrinólisis.

Las MV de monocitos pueden transportar FT y ser una fuente alterna de este 9 .

Por otro lado, las MV pueden ofrecer activadores del plasminógeno y ser una fuente de plasmina.

Este escenario muestra la importancia de las MV, su presencia se asocia con las enfermedades trombóticas arteriales y venosas que cursan con deterioro del endotelio vascular y que culminan con la formación de un trombo, así como de estados crónicos metabólicos y autoinmunes que los favorecen como son, entre otras, las enfermedades cardiovasculares y cerebrovasculares.

Nuestro grupo ha desarrollado estrógenos sustituidos en la posición 17 beta del anillo estrogénico, que en grado variable disminuyen la coagulación, la actividad de las plaquetas y la síntesis de NO, lo que favorece una vasodilatación sostenida que evita la trombosis ${ }^{10}$. Mismo se sintetizaron compuestos diméricos buame y bisebudia (compuesto dimérico de buame), que mostraron un efecto inhibidor de la agregación plaquetaria y disminución de la liberación de trifosfato de adenosina (ATP) por las plaquetas ${ }^{11}$.

Todos estos efectos inmediatos sobre la actividad de las plaquetas orientan a reconocer un efecto no genómico de estos estrógenos, sobre las membranas biológicas tanto de los organelos celulares como de la membrana celular. Este efecto se pone de manifiesto al verificar que el ATP que se libera de la agregación plaquetaria disminuye con el efecto de los aminoestrógenos, apoyando el mecanismo que disminuye la formación de MV y exosomas, ya que el ATP se encuentra dentro de los gránulos densos de las plaquetas.

También es posible reconocer una interacción principalmente en el receptor estrogénico beta de los aminoestrógenos ${ }^{12}$.

Con la intención de aportar nuevos compuestos aminoestrógenos, con actividad anticoagulante, antiagregante plaquetaria y estudiar su efecto sobre la producción de MV, sintetizamos un nuevo compuesto, tyrame, cuya estructura de alcohol amina incluye a la tiramina dentro de su molécula.

\section{Material y métodos}

\section{Reactivos}

Estrona, tiramina, tolueno anhidro, etanol absoluto, metanol absoluto, deuterocloroformo ( $\mathrm{CDCl} 3)$, dimetiIsulfóxido-d6 (DMSO-d6) y borohidruro de sodio se 
adquirieron en Sigma-Aldrich Chemical Co. ADP, epinefrina y colágena Chrono-Par".

Todos los demás reactivos empleados fueron de la máxima pureza posible.

\section{Síntesis de tyrame}

Se obtuvo ${ }^{13}$ en el Instituto de Química de la Universidad Nacional Autónoma de México (UNAM). Como se describió previamente, se empleó como disolvente tolueno anhidro, seguido de una reacción de reducción con borohidruro de sodio en metanol. Su caracterización y pureza química se determinó mediante los métodos analíticos y espectroscópicos habituales. La figura 1 muestra la síntesis de tyrame.

\section{Efecto anticoagulante de tyrame}

Los experimentos que a continuación se detallan se sometieron para su aprobación a la Comisión de Investigación y Ética de la Facultad de Medicina de la UNAM, con el protocolo número 044-2011. Se siguieron los lineamientos de empleo de animales vivos de laboratorio publicados en Guide for the Care and Use of Laboratory Animals.

El efecto anticoagulante del tyrame se determinó en sangre total empleando ratones machos cepa CD1, que se mantuvieron bajo periodos controlados de luz y obscuridad, temperatura controlada y con alimento y agua ad libidum.

Los animales se asignaron a los diferentes grupos haciendo una distribución equilibrada por su peso y el grupo al que se administró DMSO se consideró el grupo control. El tyrame se disolvió en DMSO y se administró por vía subcutánea, 0,1 y $2 \mathrm{mg} / 100 \mathrm{~g}$ de peso, con el fin de elaborar una curvas dosis-respuesta transcurridas $24 \mathrm{~h}$ de su administración.

El efecto anticoagulante en sangre total se determinó como se describe en González, et al., 20100.

\section{Efecto antiagregante plaquetario}

Se emplearon plaquetas de donadores de Banco de Sangre del Instituto Nacional de Cardiología Ignacio Chávez. En cada determinación, se empleó $9 \mathrm{ml}$ de sangre de 4 donadores. Los donadores cumplieron con los requisitos del Banco de Sangre, se recibió la sangre en tubos Vacutainer ${ }^{\natural}$ que contenían citrato de sodio $0.129 \mathrm{M}$ como anticoagulante. Las plaquetas se aislaron por centrifugación a $100 \times \mathrm{g}$ durante $1 \mathrm{~min}$, se separaron del paquete globular, $y$

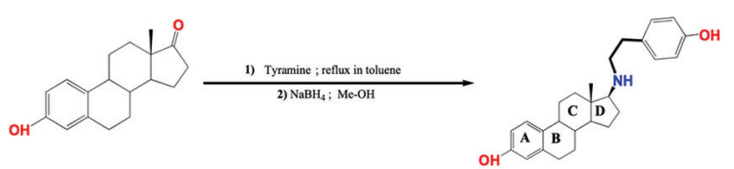

Figura 1. Síntesis de tyrame, una mezcla de estrona (540 mg, 2 $\mathrm{mM}$ ) y tiramina $(300 \mathrm{mg})$ en $350 \mathrm{ml}$ de tolueno anhidro se calentó a reflujo, con agitación, durante 48 horas, usando una trampa DeanStark. La mezcla de reacción se concentró hasta sequedad con vacío y el sólido resultante se enfrió a temperatura ambiente. El producto sólido vítreo se disolvió con metanol anhidro caliente $(350 \mathrm{ml})$ y la solución se enfrió a temperatura ambiente. Posteriormente, se adicionó borohidruro de sodio (400 mg). Después, la mezcla se calentó a reflujo a durante 20 minutos. La solución se vertió en agua con hielo y se obtuvieron cristales blancos, los cuales se filtraron y secaron. Se recristalizaron en metanol-agua y alcanzó un rendimiento de $209 \mathrm{mg}$ del producto blanco puro.

se obtuvo el plasma rico en plaquetas (PRP). El remanente de sangre se centrifugó a 250,000 x g durante $15 \mathrm{~min}$. Las plaquetas se ajustaron a $250,000 / \mu \mathrm{l}$.

La actividad antiagregante plaquetaria se determinó como se describe en González, et al., 2010¹0.

\section{Disminución de la formación de microvesículas}

Para determinar la concentración circulante de MV, se empleó la sangre de los ratones tratados con $4 \mathrm{~g} / 100 \mathrm{~g}$ del experimento de efecto anticoagulante en sangre total. La muestra se obtuvo bajo anestesia con ketamina $(50 \mathrm{mg} / \mathrm{kg})$, intramuscular, como inductor y fenobarbital $(10 \mathrm{mg} / \mathrm{kg})$ como anestésico de efecto prolongado. La muestra de sangre se obtuvo de la aorta abdominal, $2 \mathrm{ml}$, se colocó en un tubo con citrato de sodio $(0.105 \mathrm{M})$. Los ratones se sacrificaron con una sobredosis de fenobarbital.

\section{Análisis estadístico}

Los resultados se expresan como promedios \pm desviación estándar y se analizan estadísticamente por la prueba de ANOVA empleando Dunnet como prueba post hoc. Se considera un valor de $p<0.05$ como estadísticamente significativo.

\section{Resultados}

\section{Caracterización de tyrame}

La figura 2 muestra la estructura de rayos $X$ de hidrato de tyrame y los datos de los espectros masas, resonancia magnética nuclear e infrarrojo. 


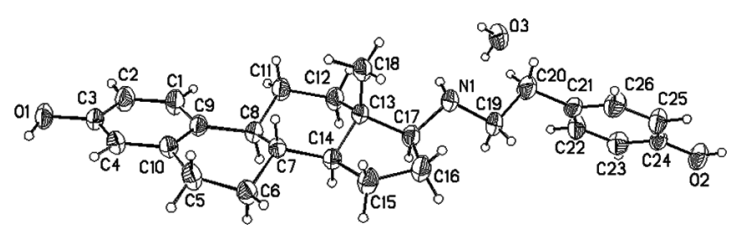

Figura 2. Tyrame [N-(3-hidroxi-1:3:5(10)-estratrien-173-il)-4-hidroxifenetilamina], rendimiento del 35\%, cristales blancos; punto de fusión $171^{\circ} \mathrm{C}$. Análisis elemental en porcentaje ( $\left.\mathrm{C}, 80.51 ; \mathrm{H}, 9.50 ; \mathrm{N}, 4.60\right)$. Calculado para $\mathrm{C}_{26} \mathrm{H}_{31} \mathrm{NO}_{2}$ (C: 80.49; $\mathrm{H}$ 9.46; $\left.\mathrm{N}: 4.69\right)$. El espectro de masas (m/z): $M^{+} 391$ (pico base 284); iR (en pastilla de $K B r$, en $\mathrm{cm}^{-1}$ ) v-O-H 3485. Resonancia magnética nuclear ${ }^{1} \mathrm{H}\left(\mathrm{CDCl}_{3}-\mathrm{DMSO}-d_{6}\right.$ en ppm), $0.70\left(\mathrm{~s}, 3 \mathrm{H}, 18 \mathrm{CH}_{3}\right), 1.16-2.25(\mathrm{~m}), 6.46\left(\mathrm{~d}, 3 \mathrm{H}, \mathrm{J}=5 \mathrm{~Hz}, \mathrm{H}_{4}\right.$, $\left.H_{22}, H_{26}\right), 6.58\left(d d, 4 H, J=5 \mathrm{~Hz}\right.$ y J $\left.=17 \mathrm{~Hz}, H_{2}, H_{4}, H_{25}, H_{23}\right), 7.04(d$, $\left.3 \mathrm{H}, \mathrm{J}=17 \mathrm{~Hz}, \mathrm{H}_{1}, \mathrm{H}_{2}, \mathrm{H}_{26}\right), 8.78\left(\mathrm{~s}, 2 \mathrm{H}, \mathrm{O}_{1}\right.$ y $\left.\mathrm{O}_{2}\right)$.

Muestra el ion molecular esperado [M] + a $391 \mathrm{~m} / \mathrm{z}$. El pico base está en $284 \mathrm{~m} / \mathrm{z}$.

El espectro infrarrojo mostró una banda de vibración, $\mathrm{v}-\mathrm{O}-\mathrm{H}$ que comienza alrededor de $3,500 \mathrm{~cm}^{-1}$, pero debido a la fuerte unión de hidrógeno, la banda es muy ancha. También están presentes las bandas correspondientes a $v-C$ alifático- $\mathrm{H}$ y $v-C$ aromático- $\mathrm{H}$. Hay una banda de alrededor de 1,607 $\mathrm{cm}^{-1}$, que se asigna a las vibraciones aromáticas $C$ - $C$ - $C$.

La estructura de rayos $X$ presenta efectos de dispersión anómala. En estado cristalino presenta varios enlaces de $H$ provenientes de moléculas de agua entre $\mathrm{O}(1)-\mathrm{H}(1 \mathrm{~A})$ del grupo $\mathrm{H}_{2} \mathrm{O}$ con el átomo de nitrógeno $N(1) \# 1$ de la molécula vecina. Existen también contactos entre $H$ con $O(1)(2.626 \AA) ; H(19)-C(2)(2.889 \AA) ; C(2)-H(16)(2.889 \AA)$ y $H(20)-H(4)(2.374 \AA$ ) de una molécula vecina.

Debido al carácter aromático del anillo $A, C(1), C(2), C(3), C(4)$, $C(10), C(9), C(5), C(8)$ y $O(1)$ deben ser coplanares. Sin embargo, el ángulo entre los planos $C(1)-C(2)-C(3)-O(1)-C(4)-C(10)-C(9)$ y $C(8)-C(9)-C(10)-C(5)$ es de $3.19(0.17)^{\circ}$. El anillo $B$ tiene una conformación de media silla. El anillo $C$ muestra una conformación en silla con sustituyentes en $C(17)$ y en $C(13)$ en posición axial. El anillo $D$ muestra una conformación 13- $\beta$ en sobre (ángulo de torsión $C(17)$ $\left.C(13)-C(14)-C(15)=45.76(0.21)^{\circ}\right)$. La geometría del anillo $D$ con la cadena lateral es trans a $C(13)$ and gauche a $C(16)$.

Las longitudes de los enlaces y los ángulos son como los reportados para otros compuestos con características similares. Los elipsoides se calculan con un $50 \%$ de probabilidad.

\section{Efecto anticoagulante en sangre total de tyrame}

La tabla 1 muestra los resultados de los tiempos de coagulación en sangre total.

\section{Efecto antiagregante plaquetario de tyrame}

La tabla 2 muestra el efecto inhibitorio de la agregación plaquetaria.

\section{Efecto inhibidor de la formación de MV}

La figura 3 muestra que el efecto inhibidor de la formación de MV de tyrame.
Tabla 1. Los compuestos se disolvieron en dimetilsulfóxido (DMSO) y se calcularon para $100 \mathrm{~g}$ de peso de ratón; se administraron por vía subcutánea. A las 24 h se determinó el tiempo de coagulación en sangre total, se dilató la vena dorsal del ratón (colocando la cola en baño maría, a $37^{\circ} \mathrm{C}$, durante $3 \mathrm{~min}$ ). Posteriormente, se hizo un corte trasversal para obtener $\pm 25 \mu \mathrm{l}$ de sangre total, la cual fue colectada en un capilar previamente marcado en dos extremos. Se permite desplazar la sangre entre las dos marcas en un equipo que limita el movimiento a $60^{\circ}$. Cuando inicia el llenado del capilar se activa un cronómetro, cuando se detiene el desplazamiento de la sangre se detiene el cronómetro y registra, en segundos

\begin{tabular}{|l|c|r|}
\hline Compuesto & Dosis & Tiempo en s \\
\hline Tyrame & DMSO & $124.3 \pm 19$ \\
\hline $1 \mathrm{mg} / 100 \mathrm{~g}$ & $162.7 \pm 11.6$ \\
\hline $2 \mathrm{mg} / 100 \mathrm{~g}$ & $191.0 \pm 8.4^{*}$ \\
\hline
\end{tabular}

${ }^{*} \mathrm{p} \leq 0.05$ se considera estadísticamente significativo, ANOVA con post hoc Dunnett, SPSS $v 21.0$.

Tabla 2. En cada determinación se colocaron $500 \mu \mathrm{l}$ de plasma rico en plaquetas en un tubo de agregación plaquetaria, se incubaron durante $5 \mathrm{~min}$ a $37{ }^{\circ} \mathrm{C}$, posteriormente se adicionaron $10 \mu \mathrm{l}$ de tyrame a diferentes concentraciones (5-500 mM) e inmediatamente después el agente agonista, $\mathrm{ADP} 10 \mu \mathrm{M}$, epinefrina $10 \mu \mathrm{M}$ o colágena $2 \mathrm{~g} / \mathrm{ml}$. Equipo Lumi-Aggregometer (Model $560 \mathrm{CA}$ acoplado a un programa Model 810 AGGRO/LINK Chrono-log, Havertown, PA, EE.UU.). El agente agonista activa a las plaquetas induciendo un cambio de forma que inmediatamente impide brevemente el paso de luz, a medida que la respuesta se vuelve irreversible el trazo se modifica hasta alcanzar una meseta. El porcentaje de agregación plaquetaria alcanzado por el dimetilsulfóxido (DMSO) se considera un $100 \%$ y los resultados se expresan en porcentaje con respecto al grupo control. Los resultados se expresan en promedio \pm desviación estándar. Los resultados de agregación plaquetaria que se obtienen empleando el disolvente DMSO (grupo control) y el agente inductor se consideran como el $100 \%$ de agregación y $0 \%$ de inhibición. Los resultados obtenidos con las diferentes concentraciones de tyrame de cada agente agonista se calcularon mediante el porcentaje de agregación con respecto al control y se obtiene el porcentaje de inhibición. Por ejemplo, para un porcentaje de inhibición del $78.4 \%$ significa que el porcentaje de agregación fue del $21.6 \%$. Los agentes inductores se preparan según las indicaciones del fabricante Sigma

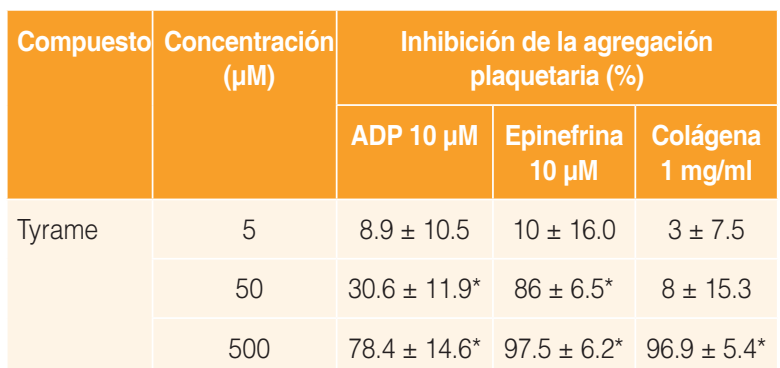

${ }^{*} \mathrm{p}<0.05$ se considera estadísticamente significativo, ANOVA con post hoc Dunnett, SPSS $v 21.0$.

\section{Conclusiones}

Los resultados muestran que el tyrame tiene un discreto efecto anticoagulante a las $24 \mathrm{~h}$ de su 


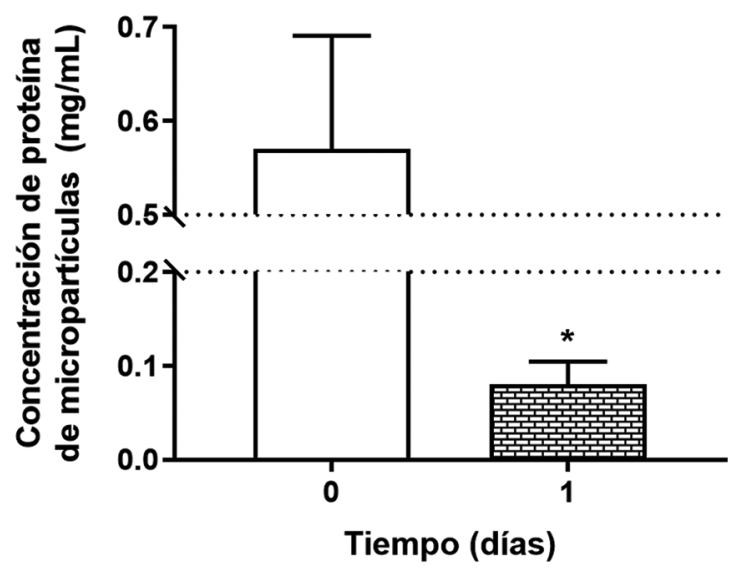

Figura 3. Las MV se aislaron mediante centrifugación diferencial, la muestra sanguínea citratada $(0.105 \mathrm{M})$, se centrifugó dos veces (1,500 x g por 15 min y 13,000 x g por 2 min) para obtener el plasma pobre en plaquetas. $500 \mu$ / de muestra se centrifugaron $(20,000 \times g$ por $90 \mathrm{~min}$ a $4^{\circ} \mathrm{C}$ ), las $\mathrm{MV}$ fueron lavadas y resuspendidas con amortiguador HEPES (10 mM NaCl, 140 mM HEPES; pH 7.4). Las MV se rompieron mediante choque térmico y la concentración de proteína se determinó a $280 \mathrm{~nm}$ en un espectrofotómetro.

Los resultados se expresan como concentración de proteína.

La concentración de MV disminuyó seis veces a las $24 \mathrm{~h}$ de tratamiento ( $4 \mathrm{~g} / 100 \mathrm{mg}$ ), el resultado se expresa en $\mathrm{mg}$ de proteína/ml, lectura en un espectrofotómetro a $280 \mathrm{~nm}$. Se considera que al formarse las MV se organizan en su estructura los receptores, proteínas, $A D N, A R N$ miARN y otras moléculas presentes en la célula original.

administración, se observa un comportamiento similar a los mostrados por otros aminoestrógenos. Sin embargo, su efecto antiagregante plaquetario es superior sobre todo cuando se emplea colágena como agente inductor.

Este efecto antiagregante plaquetario se refleja en la marcada disminución de MV circulantes.

Este efecto resalta y pone en posible ventaja el uso de tyrame como un estrógeno sin riesgo trombótico por disminuir la formación de MV, lo que sería de utilidad terapéutica en diferentes tipos de cáncer ${ }^{14}$, trastornos cardiovasculare ${ }^{15}$ y enfermedades autoinmunes ${ }^{16}$ en donde las MV tienen un papel importante en su fisiopatología.

\section{Financiamiento}

El proyecto recibió el apoyo presupuestario del Departamento de Farmacología, Facultad de Medicina, UNAM y del Departamento de Biología Molecular, Instituto Nacional de Cardiología.

\section{Conflicto de intereses}

Los autores declaran no tener conflicto de intereses.

\section{Responsabilidades éticas}

Protección de personas y animales. Los autores declaran que los procedimientos seguidos se conformaron a las normas éticas del comité de experimentación humana responsable y de acuerdo con la Asociación Médica Mundial y la Declaración de Helsinki.

Confidencialidad de los datos. Los autores declaran que han seguido los protocolos de su centro de trabajo sobre la publicación de datos de pacientes.

Derecho a la privacidad y consentimiento informado. Los autores declaran que en este artículo no aparecen datos de pacientes.

\section{Bibliografía}

1. Mosca L, Collins P, Herrington DM, Mendelsohn ME, Pasternak RC, Robertson RM, et al. Hormone replacement therapy and cardiovascular disease: A statement for healthcare professionals from The American Heart Association. Circulation. 2001;104:499-503.

2. Writing Group for the Women's Health Initiative Investigators. Risk and benefits of estrogen plus progestin in healthy postmenopausal women: principal results from the Women's Health Initiative randomized controlled trial. J Am Med Assoc. 2002;288:321-33.

3. Grady D, Herrington D, Bittner V, Blumenthal R, Davidson M, Hlatky M, et al. Cardiovascular disease outcomes during 6.8 years of hormone therapy: Heart and Estrogen/progestin Replacement Study follow-up (HERS II). JAMA. 2002;288(1):49.

4. Martinelli I, Taioli E, Buciarelli P, Akhavan S, Manucci PM. Interaction between the G20210A mutation of the prothrombin gene and oral contraceptive use in deep vein trombosis. Arterioscler Thromb Vasc Biol. 1999;19:700-3.

5. Rosendaal FR, Koster T, Vandenbroucke JP, Reitsma PH. High risk of thrombosis in patients homozygous for factor $V$ Leiden (activated protein C resistance). Blood. 1995;85:1504-8.

6. Tchaikovski SN, Rosing J. Mechanisms of estrogen-induced venous thromboembolism. Thromb Res. 2010;126(1):5-11.

7. Furie B, Furie BC. Thrombus formation in vivo. J Clin Invest. 2005;115:3355-62

8. Dejouvencel T, Doeuvre L, Lacroix R, Plawinski L, Dignat-George F, Lijnen $\mathrm{H}$, et al. Fibrinolytic cross-talk: a new mechanism for plasmin formation. Blood. 2010;115:2048-56.

9. Halvorsen H, Olsen JO, Østerud B. Granulocytes enhance LPS-induced tissue factor activity in monocytes via an interaction with platelets. J Leukoc Biol. 1993:54:275-82.

10. González G, Alvarado-Vasquez N, Fernández-G JM, Cruz-Robles D, del Valle $L$, Pinzón $E$, et al. The antithrombotic effect of the aminoestrogen prolame (N-(3-hydroxy-1,3,5(10)-estratrien-17B-yl)-3-hydroxypropylamine) is linked to an increase in nitric oxide production by platelets and endothelial cells. Atherosclerosis. 2010;208:62-8.

11. Flores-García M, Fernández-G JM, León-Martínez M, Hernández-Ortega S, Pérez-Méndez $\mathrm{O}$, Correa-Basurto $\mathrm{J}$, et al. The structures and inhibitory effects of Buame [N-(3-hydroxy-1,3,5(10)-estratrien-17 $\beta$-yl)-butylamine] and Diebud [N,N'-bis-(3-hydroxy-1,3,5(10)-estratrien-173-yl)-1,4-butanediamine] on platelet aggregation. Steroids. 2012;77:512-20.

12. Lemini C, Rubio-Póo C, Franco Y, Jaimez R, Avila ME, Medina M, et al In vivo profile of the anticoagulant effect of 17ß-amino-1,3,5(10)estratrien-3-ol. Eur J Pharmacol. 2013;700:210-6.

13. Fernández-G JM, Rubio-Arroyo MF, Soriano-García M, Toscano RA Pérez-Cesar M del C, Rubio-Póo C, et al. Synthesis and molecular structure of Prolame, [N-(3-hydroxy-1,3,5(10)-estratrien-17 $\beta$-yl)-3-hydroxypropylamine; an amino estrogen with prolonged anticoagulant and brief estrogenic effects. Steroids. 1985;45:151-7.

14. Ender F, Freund A, Quecke T, Steidel C, Zamzow P, von Bubnoff N, et al. Tissue factor activity on microvesicles from cancer patients. J Cancer Res Clin Oncol. 2020;146:467-75

15. Chiva-Blanch G, Badimon L. Cross-talk between lipoproteins and inflammation: The role of microvesicles. J Clin Med. 2019:8(12):2059.

16. Wu X, Liu Y, Wei W, Liu ML. Extracellular vesicles in autoimmune vasculitis - Little dirts light the fire in blood vessels. Autoimmun Rev. 2019;18:593-606. 